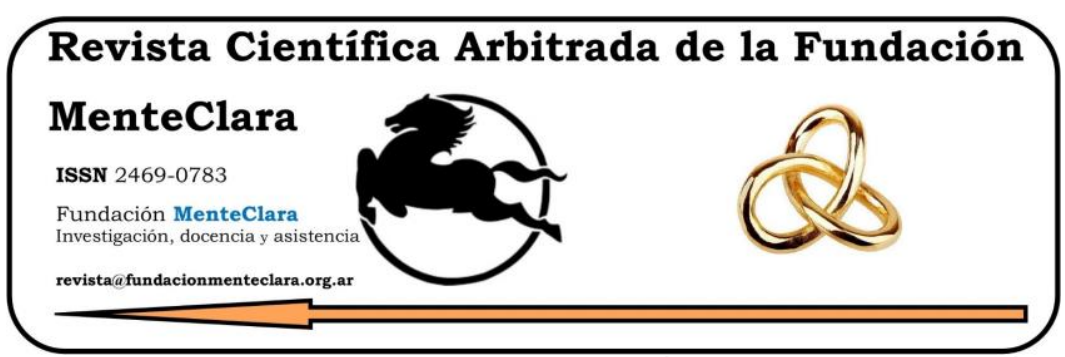

Artículos atravesados por (o cuestionando) la idea del sujeto -y su género- como una construcción psicobiológica de la cultura. Articles driven by (or questioning) the idea of the subject -and their gender- as a cultural psychobiological construction.

Vol. 6 (2021), enero-diciembre ISSN 2469-0783

https: / / datahub.io/dataset/2021-6-e236

\title{
RELACIÓN ENTRE FELICIDAD Y ESTRATEGIAS DE AFRONTAMIENTO
}

\section{CORRELATION BETWEEN HAPPINES AND COPING STRATEGIES}

Iván Daniel González ivan.gonzalez@uflounivesidad.edu.ar

Estudiante de Psicología, Cátedra Psicología de la Salud, Universidad de Flores, Argentina.

Cómo citar este artículo / Citation: González, I. (2021). Relación entre Felicidad y Estrate gias de Afrontamiento. Revista Científica Arbitrada de la Fundación MenteClara, Vol. 6 (236). DOI: https://doi.org/ 10.32351/rca.v6.236

Copyright: (C) 2021 RCAFMC. Este artículo de acceso abierto es distribuido bajo los términos de la licencia Creative Commons Attribution 4.0 International License (CC BY 4.0). Recibido: 10/07/2021. Aceptado: 16/07/2021 Publicación online: 21/07/2021

Conflicto de intereses: Ninguno que declarar.

\section{Resumen}

En la presente investigación se ha realizado una revisión narrativa en la cual se trabajaron las variables Felicidad y Estrategias de Afrontamiento, permitiendo establecer que el empleo de las estrategias adecuadas puede conducir al sujeto hacia la Felicidad. Para esto se ha definido a la Felicidad como un constructo de carácter unidimensional y sin especificación constituyente, con una conceptualización de origen filosófico (Eudaimonía y Hedonismo) que pasaría, posteriormente, al campo de la Psicología como una sumatoria de beneficios para la salud mentaly física. Por otro lado, la segunda variable, Estrategias de Afrontamiento, se conceptualizó como el conjunto de respuestas o comportamientos hacia demandas tanto evolutivas como del ambiente inmediato, siendo estas Adaptativas (referidas a la resolución de la demanda mediante medidas constructivas) o Inadaptativas (orientadas a conductas de carácter de riesgo y/o evasión de la problemática). Se determinó que el desarrollo de la inteligencia emocional permitía un mejor manejo de las emociones a la hora de elegir una estrategia, permitiéndole al sujeto apreciar mejor el obstáculo, gracias a la 
naturaleza interventora del afrontamiento; de esta manera se concluyó que las Estrategias de Afrontamiento repercuten sobre la Felicidad de las personas, generando beneficios tanto mentales como biológicos.

\begin{abstract}
During this investigation it has been developed a narrative review about the Happiness and Coping Strategies variables, allowing us to establish that the implementation of coping may allow any subject to a state of happyness. For this mean, the concept of Happyness has been defined as an unidimensional construct with any constitutive especification that departs from a philosophichal conceptualization (Eudaimonia and Hedonism), which eventually would arrive to the psichological field as a sum of benefits for the mental and phisical health. On the other side, the second variable, Coping Strategies, was described as a set of answers and manners oriented to evolutive and ambiental demands, being classified as either Adaptive (meant for the resolution of taks via constructive means) or Maladaptive (those which have an orientation towards risk or evasive copping). It was determined that an appropriate development about emotional intelligence would allow the subject to have a more efficient control of his/her emotions when opting for coping strategy, making it easier for the subject to fully comprehend the task at hand due to the intervenering nature of the coping itself, reaching to the conclusion that Coping Strategies affects people Happyness, generating benefits for their mental and biological status.
\end{abstract}

Palabras Claves: Felicidad; Estrategias de Afrontamiento; Adaptativas; Inadaptativas; Bienestar

Keyw ords: Happiness; Coping Strategies; Adaptive; Maladaptive; Well Being 


\section{Introducción}

La Felicidad representa uno de los elementos de mayor importancia en el campo de la Psicología Positivista y su definición ha estado bajo la lupa de múltiples corrientes filosóficas para luego tomar su lugar en la Psicología (Moyano Díaz, 2018).

Al margen de lo anterior mencionado, las metodologias a la hora de abordar la búsqueda de la felicidad están sujetas a la variable de que cada ser humano es diferente y por ende, opera de distintas maneras. El éxito o fracaso frente a un obstáculo dependerá, entonces, de cómo el sujeto proceda ante la situación y de qué Estrategias de Afrontamiento hará uso (Salavera Bordás \& Usán Supervia, 2017).

En el presente trabajo se buscara desarrollar con mejor detalle las conceptualizaciones que permiten una mejor comprensión de la Felicidad y cómo esta afecta la salud mental, la importancia de las adecuadas Estrategias de afrontamiento frente a un obstáculo, como no todas llevan a resultados fructíferos y la buena vinculación existente entre la Felicidad y las Estrategias.

\section{Felicidad}

El concepto de Felicidad como tal no ha sido consensuado de manera definitiva, pero se ha podido tomar en consideración cómo unidimensional y sin especificación de que es lo que lo constituye (Moyano Díaz, 2018). Esto se debe, muy probablemente, a que su relevancia ha sido tomada en cuenta en el campo de la Psicología de manera reciente, más precisamente a mediados del siglo XX, cuando el afán por el análisis científico del bienestar comenzó a surgir (Dominguez Bolaños \& Cruz Ibarra, 2017). El enfoque para la Psicología residía en las patologias del comportamiento y todo lo que involucre cuestiones 
negativas (Gabini, 2019), siendo Martin Seligman una de las figuras discernientes de mayor relevancia quien proponía el estudio de lo positivo desde tres ángulos; el análisis de la experiencia positiva, estudiar las fortalezas económicas y estudiar qué características rigen sobre las organizaciones positivas (Dominguez Bolaños \& Cruz Ibarra, 2017).

La conceptualización de la Felicidad y las maneras para poder alcanzarla tienen sus orígenes en perspectivas filosóficas tales como la Eudaimonía y la Hedonismo, siendo las afirmaciones de esta última las que pasarian a tener mayor peso en la Psicología a futuro (Moyano Díaz, 2018). Esta corriente consideraba que las bases para el alcance de la felicidad estaban centradas en tres máximas; alcanzar la mayor sumatoria posible de momentos placenteros, la inmediata satisfacción de los deseos y en la evitación de lo que pueda provocar dolor (Dominguez Bolaños \& Cruz Ibarra, 2017). Por otro lado, partiendo desde la corriente de la Eudaimonia (término proveniente de los pensadores de corte estoico), se propone que la felicidad puede ser alcanzada mediante el desarrollo del potencial humano a través del virtuosismo, una dotación de sentido para la vida y el desarrollo de la espiritualidad. (Moyano Díaz, 2018).

La importancia de la felicidad para la salud mental queda en evidencia frente a los beneficios que el individuo experimenta, tales como el aumento en la productividad laboral, mayor autocontrol y rendimiento, mejoras en los estados de salud físico y mental, una mayor longevidad y mejores relaciones interpersonales (Gabini, 2019), una mayor resistencia al dolor, y además guarda una relación con un mejor desarrollo económico y empresarial (Dominguez Bolaños \& Cruz Ibarra, 2017). Es de destacar, que en este último concepto se observa una mayor escala de felicidad en los emprendedores de pequeños emprendimientos por encima de los que dirigen emprendimientos de mayor calibre (Gabini, 
2019). Otros de los beneficios que el individuo experimenta a través de una mayor felicidad, son: la disposición de una familia que presente seguridad, unión, apoyo y protección, la orientación al logro de metas y objetivos propuestos, el desarrollo del optimismo (evaluación retrospectiva positiva) y el equilibrio psicológico (Moyano Díaz, 2018).

Como nota aparte, podemos hacer mención a una de las muchas potenciales definiciones para la Felicidad, dilucidada a partir de encuestas a múltiples individuos:

Un estado más o menos transitorio de equilibrio, relativo a condiciones, resultados o logros individuales o grupales favorables o reconfortantes para la persona, generalmente asociados a una sensación de satisfacción y bienestar. Este estado surge con base a la estabilidad de ciertos elementos relevantes para la persona, de seguridad y sostén básico en lo económico (trabajo) y familiar, que le posibilitan dedicarse con libertad a actividades que implican posibilidades de goce, desarrollo y plenitud (Moyano Díaz, 2018).

\section{Estrategias de Afrontamiento}

Las estrategias de afrontamiento se definen como la respuesta generada a partir de patrones de comportamiento con el objetivo de satisfacer las demandas provenientes del propio proceso evolutivo, como del contexto social inmediato (Uribe Urzola et al., 2018).

A la hora de clasificar las Estrategias de Afrontamiento es posible proceder con dos tipos de denominaciones: adaptativas y no adaptativas o inadaptativas -posibles respuestas ante sucesos potencialmente traumáticos-, presentando características a nivel conductual, tales como la evitación, el aislamiento o rechazo de ayuda psicológica, conductas de riesgo o abuso de estupefacientes y/o alcohol en el caso de las 
inadaptativas, mientras que la búsqueda de ayuda de carácter terapéutico, social y familiar, conductas de carácter altruista, actividades orientadas a la gratificación sin riesgos mayores y la exposición a estímulos similares a eventos traumáticos son características de las Estrategias de Afrontamiento adaptativas (Echuburúa \& Amor, 2019).

Es también importante destacar que las estrategias de afrontamiento se encuentran presentes no solo a nivel conductual sino también a nivel cognitivo, como ser en estrategias tales como la atención selectiva a eventos traumáticos en el caso de las inadaptativas y focalización atencional en situaciones positivas a presente y/o futuro para las adaptativas (Echuburúa \& Amor, 2019).

Según diversos estudios llevados a cabo entre universitarios de distintos niveles; se concluyó que el Afrontamiento por Aproximación Cognitiva -preparación mental previa a un evento estresante- era la estrategia predilecta por los estudiantes universitarios de la carrera de Psicologia, estando también presentes estrategias de evitación, reevaluación positiva y/o búsqueda de apoyo, tanto por parte de estudiantes de la mencionada carrera como para estudiantes de carreras referidas a otros aspectos de la salud (Sicre \& Casari, 2019).

Existen tres estilos de afrontamiento en pos de la adaptación que guardan relación con el género de quien las escoge; contamos con el estilo enfocado a la resolución individual de la situación (más acentuado en los varones), el estilo referido a la búsqueda de apoyo (presente en ambos géneros pero con más visibilidad en las mujeres) y el estilo improductivo, el cual no produce bienestar emocional ni efectos positivos, como por ejemplo la evitación -demostrando una carencia de capacidad empírica a la hora de solventar un problema, con frecuencia hallado en los varones(Uribe Urzola et al., 2018). 
El resultado de una estrategia de afrontamiento, sea este fallido o exitoso, no necesariamente implica que existan estrategias sea más óptima que otra; la evaluación de sus efectos debe estar sujeta a varios factores como ser la disposición de ambiente, el momento vital de la persona o el fin por el cual se persigue determinada conducta. (Uribe Urzola et al., 2018)

\section{Felicidad y Estrategias de Afrontamiento}

Un buen punto de partida en pos de la vinculación entre las variables Felicidad y Estrategias de Afrontamiento, sería definiendo "inteligencia emocional", concepto concebido la capacidad para generar y reconocer emociones propias y expresarlas adecuadamente, además de poder percibir las ajenas y promover el crecimiento emocional e intelectual (Millán-Franco et al., 2020). La importancia de la Inteligencia Emocional yace en el desarrollo de habilidades emocionales (mediante, por ejemplo, cursos específicos o ayuda por parte de organizaciones sociales) que permitirian dirimir estrategias de afrontamiento de mayor eficaciay, por consiguiente, alcanzar un mejor desarrolla de la felicidad y el bienestar propio (Millán-Franco et al., 2020). El afrontamiento juega un rol interventor en las emociones, tanto en la atención, regulación y comprensión de las mismas, lo que permite -en la mayoría de los casosresolver los problemas, obstáculos y tareas, focalizándose en la raíz de los mismas/os. (Salavera Bordás \& Usán Supervía, 2017).

Dentro de los estudios que vinculan las Estrategias de Afrontamiento con la Felicidad (Moyano-Díaz, 2017), se ha podido determinar que estrategias vinculadas con la salud mental como ser la aceptación, la distracción, la modificación de la situación -por medio de vía directa-, la baja supresión, la re-evaluación positiva o la expresión regulada guardan 
relación con elementos tales como la afectividad positiva y el bienestar. Otro estudio (Uribe Urzola et al., 2018), llevado a cabo entre 1402 estudiantes de secundaria -entre las edades de 12 a 14 años-de ambos géneros -711 chicos y 691 chicas-, presentó resultados donde resaltaron variables tales como; la edad, cuya influencia quedó demostrada debido a la presencia del optimismo en los participantes de edad más temprana -adolescencia- en comparación al pesimismo más acentuado en los adultos nacido a partir de los fracasos acarreados hasta ese punto de sus vidas; el sentido de pertenencia y la búsqueda de ayuda social mediante el apoyo de otros en pos del alcance de la superación de la meta; el género, dado que se determinó que las estrategias adoptadas por los varones se inclinaban más por el lado del distanciamiento, la evitación, tratar la dificultad de manera más personal como también hacer búsqueda de apoyo social en personal profesional, mientras que las chicas hicieron un mayor hincapié en la búsqueda de apoyo social y en la reducción de la tensión.

\section{Conclusión}

En dicha investigación se ha podido desarrollar la relación entre la Felicidad y las Estrategias de Afrontamiento mediante distintos estudios con buen grado de fiabilidad y rigor científico, en donde se ha notado la presente influencia de la psicología positivista en la mayoría de los casos. Además, se ha podido elaborar acerca de la Felicidad y las Estrategias de Afrontamiento con un mayor grado de profundidad-dentro de las disponibilidades que este trabajo permite-, implicando un desarrollo de los distintos tipos de estrategias que pueden ser empleadas -dependiendo su selección o preferencia por parte de los individuos a sus propias habilidades y virtudes- en pos de alcanzar un grado de gratificación y felicidad subjetiva -estrategias adaptativas- y, en casos contrarios, 
estrategias que conducen a resultados alejados de estos resultados y que están más orientados hacia conductas perjudiciales para el propio bienestar-estrategias inadaptativas-. Es importante no dejar de remarcar que la elección de una estrategia para proceder ante algún obstáculo como ser el estrés para los estudiantes, eventos traumáticos o injusticias laborales- dependerá en las características, competencias y habilidades de la persona en cuestión, además de élámbito social en el que la persona se encuentre y con la buena -o mala- relación que pueda guardar para con quienes comparte ese ámbito social -familia y amistades-, dado que su apoyo, consejo y contención influencian en su toma de decisiones.

En conclusión, el empleo de Estrategias de Afrontamiento y su desarrollo repercuten en el bienestar de las personas y en su Felicidad, con toda la caterva de variantes posibles involucradas en ese proceso y que están presentes en aspectos tanto sociales como biológicos. 


\section{Referencias}

Dominguez Bolaños, R., \& Cruz Ibarra, E. (2017). La psicología positiva: Un nuevo enfoque para el estudio de la felicidad. Razón y Palababra, 660-679.

Echuburúa, E., \& Amor, P. (2019). Traumatic Memories: Adaptive and Maladaptive Coping Strategies. Terapia psicológica, 71-80.

Gabini, S. (2019). Felicidad en el trabajo: Breve actualizacion desde la psicologia positiva. Revista de Psicología, 69-75.

Millán-Franco et al., M. (2020). Inteligencia emocional y felicidad subjetiva en estudiantes de Trabajo Social (Emotional intelligence and subjective happiness in social work students). Alternativas. Cuadernos de Trabajo Social, 117-132.

Moyano Díaz et al., E. (2018). Escala de Felicidad para Adultos (EFPA). Terapia psicológica, 37-49.

Moyano-Díaz et al., E. (2017). Afrontamiento, salud mental y felicidad bajo huelga y ocupación universitaria. Arquivos brasileiros de Psicología, 153-167.

Salavera Bordás, C., \& Usán Supervía, P. (2017). Repercusión de las estrategias de afrontamiento de estrés en la felicidad de los alumnos de Secundaria. Revista Electrónica Interuniversitaria de Formación del Profesorado, 65-77.

Sicre, E., \& Casari, L. (2019). Estrategias de afrontamiento en estudiantes de Psicología. Revista de Psicología, 61-72.

Uribe Urzola et al. (2018). La importancia de las estrategias de afrontamiento en el bienestar psicológico en una muestra escolarizada de adolescentes. Psicogente, 440-457. 